\title{
Non-Equilibrium Phase Behavior of Diblock Copolymer Melts and Binary Blends in the Intermediate Segregation Regime
}

\author{
PAUL M. LIPIC, ${ }^{1, *}$ FRANK S. BATES, ${ }^{1}$ MARK W. MATSEN ${ }^{2}$ \\ ${ }^{1}$ Department of Chemical Engineering and Materials Science, University of Minnesota, Minneapolis, Minnesota 55455 \\ ${ }^{2}$ Polymer Science Centre, University of Reading, Whiteknights, Reading RG6 6AF, United Kingdom
}

Received 30 November 1998; revised 23 April 1999; accepted 26 April 1999

\begin{abstract}
The phase behavior of intermediately segregated ( $\chi N=45$ ) poly(ethylene)poly(ethylethylene) (PE-PEE) diblock copolymers and PE-PEE binary blends are characterized using transmission electron microscopy and small-angle X-ray scattering. Surprisingly, the preparation-dependent, nonequilibrium phase behavior can be overwhelming even at this degree of segregation. A pure diblock with a poly(ethylene) volume fraction of $f_{\mathrm{PE}}=0.46$ exhibited coexisting lamellae and perforated layers when prepared using a precipitation technique, but contained only the lamellar morphology when solvent cast. This preparation dependence was more dramatic in binary diblock copolymer blends with average compositions of $\left\langle f_{\mathrm{PE}}\right\rangle=0.44,0.46$, and 0.48 . Precipitated blends exhibited a microphase separated structure that was disordered and bicontinuous; however, solvent cast samples exhibited either a cylindrical, coexisting cylindrical and lamellar, or lamellar morphology. This nonequilibrium behavior is attributed to the high degree of segregation and the proximity to the cylinder/lamellae phase boundary. (C) 1999 John Wiley \& Sons, Inc. J Polym Sci B: Polym Phys 37: 2229-2238, 1999
\end{abstract}

Keywords: transmission electron microscopy; small angle X-ray scattering; solvent casting; precipitation; preparation dependence; blends

\section{INTRODUCTION}

Diblock copolymers have generated much experimental and theoretical interest because of their rich microstructured phase behavior. When the incompatibility of the two chemically distinct blocks becomes sufficient, a disordered melt will microphase separate producing one of several ordered morphologies. This order-disorder transition (ODT) generally occurs when $\chi N \approx 10$, where $\chi$ is the classical segment-segment inter-

* Present address: The Procter and Gamble Company, Cincinnati, Ohio 45224

Correspondence to: F. S. Bates (E-mail: jlk6@po.cwru.edu) Journal of Polymer Science: Part B: Polymer Physics, Vol. 37, 2229-2238 (1999) ( 1999 John Wiley \& Sons, Inc. $\quad$ CCC 0887-6266/99/162229-10 action parameter between the two blocks and $N$ is the overall degree of polymerization. ${ }^{1,2}$ Almost immediately after the ODT, the melt enters the intermediate segregation regime, where the microdomains become well developed requiring a large number of Fourier harmonics to describe their concentration profile. ${ }^{1}$ Once $\chi N$ exceeds about 50, the microdomains become virtually pure, the interfaces become narrow, and the melt enters the so-called strong segregation regime. ${ }^{1}$ In this regime, the domain spacing is expected to scale as $d \sim \chi^{1 / 6} N^{2 / 3}$. Recent experiments ${ }^{3}$ have largely focused on the weak to intermediate segregation regime near the ODT, partly because nonequilibrium effects are minimal. However, a considerable wealth of research has been accumulated over the past three decades on highly seg- 
regated block copolymer melts far from the ODT where nonequilibrium effects become much more important.

In addition to $\chi N$, an important parameter controlling phase behavior is the volume fraction of one of the blocks, $f$. It is primarily responsible for selecting the domain geometry (i.e., spheres, cylinders, lamellae) of an ordered melt. Changing the value of $f$ requires the synthesis of a new block copolymer, which makes mapping the phase behavior a very labor intensive task. Fortunately, the one-component approximation provides a simple method of controlling $f$ by mimicking a neat diblock melt with a binary diblock blend. ${ }^{4-6}$ According to this approximation, the blend behaves equivalently to a neat diblock melt with $f$ equal to the volume averaged composition $\langle f\rangle$ of the blend, which can easily be varied. However, caution must be used when implementing this approximation; the two diblock species must be of similar size and composition. A mismatch in size can shift
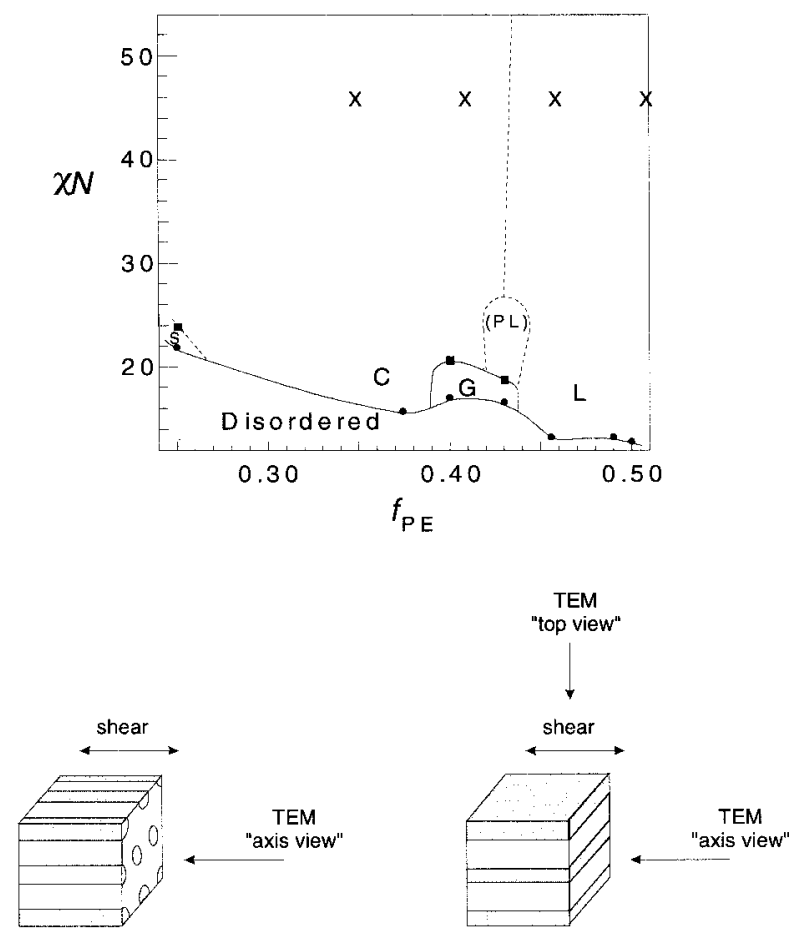

Figure 1. Experimentally determined PE-PEE phase diagram near the ODT ( $\mathrm{S}=$ body-centered cubic packed spheres, $\mathrm{C}=$ hexagonally packed cylinders, $\mathrm{G}$ = bicontinuous cubic gyroid, $\mathrm{L}=$ lamellae, $(\mathrm{PL})=$ tentative assignment of perforated lamellae). ${ }^{4}$ The locations of the four diblocks in this study are denoted by Xs where $N=1500$ and $\chi \cong 0.03 .^{2}$ The TEM viewing directions for cylinders and lamellae are illustrated at the bottom. the phase boundaries, and large differences in composition can produce significant two-phase coexistence regions. ${ }^{5}$ Moreover, nonequilibrium effects may be amplified due to the presence of two or more populations of compositionally distinct molecules that can segregate locally thereby stabilizing metastable morphologies.

This paper investigates poly(ethylene)-poly(ethylethylene) (PE-PEE) diblock copolymers deep in the intermediate segregation regime $(\chi N$ $=45$ ). Because the interaction between $\mathrm{PE}$ and $\mathrm{PEE}$ is relatively small $\left(\chi \cong 0.03\right.$ at $150^{\circ} \mathrm{C}$ based on a four-carbon repeat unit), ${ }^{2}$ this requires relatively high molecular weight diblock copolymers $\left(M_{n} \cong 8 \times 10^{4} \mathrm{~g} / \mathrm{mole}\right)$. Our study focuses on compositions near the cylinder/lamellae boundary, where perforated layers and cubic bicontinuous gyroid structures have been previously identified at weaker segregations (Fig. 1). ${ }^{4}$ Small-angle X-ray scattering (SAXS) and transmission electron microscopy (TEM) techniques are used to characterize the phase behavior for both pure diblock copolymers and binary blends. By examining the morphologies produced by different preparation methods, we assess the importance of nonequilibrium effects. Remarkably high levels of metastability are experienced even though the degree of segregation is not particularly large.

\section{EXPERIMENTAL}

\section{Block Copolymer Synthesis}

The four PE-PEE diblock copolymers used for this study (Table I) were synthesized using a previously reported method. ${ }^{2}$ Anionically polymerized poly(1,4-butadiene)-poly(1,2-butadiene) was saturated with deuterium to obtain PE-PEE. Poly(ethylene) weight fractions were calculated based on the reaction stoichiometry (the polymerizations yielded $>99 \%$ recovered polymer), and converted to volume fractions, $f_{\mathrm{PE}}$, using the reported amorphous densities for $\mathrm{PE}$ and PEE. ${ }^{8}$ The molecular weight and number average polydispersity were measured using a Waters 150C GPC at $80^{\circ} \mathrm{C}$ with 1,2,4-trichlorobenzene (Aldrich, stabilized with Irganox (Ciba-Geigy)) as the solvent and carrier fluid and based on calibration with poly(styrene) (PS) standards (the results are listed in Table I). The actual molecular weights for these polymers are significantly lower than those determined by GPC due to differences in the hydrodynamic volumes of PE, PEE, and PS at 
Table I. Molecular Characteristics of Pure Diblock Copolymers

\begin{tabular}{|c|c|c|c|c|c|c|}
\hline \multirow[b]{2}{*}{ Sample } & \multirow[b]{2}{*}{$f_{\mathrm{PE}}{ }^{\mathrm{a}}$} & \multirow[b]{2}{*}{$\begin{array}{c}M_{n}^{\mathrm{b}}(\mathrm{GPC}) \\
(\mathrm{kg} / \mathrm{mol})\end{array}$} & \multirow[b]{2}{*}{$M_{w} / M_{n}^{\mathrm{b}}$} & \multirow[b]{2}{*}{$\begin{array}{c}M_{n}(\text { corrected })^{\mathrm{c}} \\
(\mathrm{kg} / \mathrm{mol})\end{array}$} & \multicolumn{2}{|c|}{ Phases $^{\mathrm{d}}$} \\
\hline & & & & & Precipitated & $\begin{array}{l}\text { Solvent } \\
\text { Cast }\end{array}$ \\
\hline PE-PEE-21 & 0.35 & 133 & 1.07 & 77.1 & $\mathrm{C}$ & $\mathrm{C}$ \\
\hline PE-PEE-23 & 0.41 & 148 & 1.08 & 84.4 & $\mathrm{C}$ & $\mathrm{C}$ \\
\hline PE-PEE-20 & 0.46 & 145 & 1.08 & 79.8 & $\mathrm{~L} / \mathrm{PL}$ & $\mathrm{L}$ \\
\hline PE-PEE-22 & 0.51 & 167 & 1.08 & 90.2 & $\mathrm{~L}$ & $\mathrm{~L}$ \\
\hline
\end{tabular}

equal molecular weight under the measurement conditions. We have estimated the true $M_{n}$ values based on the universal calibration concept which relates elution volume to the product $[\eta] M$ where the intrinsic viscosity is given by the Mark-Houwink relationship, $[\eta]=K M^{a}$. Since 1,2,4-trichlorobenzene is a good solvent for PE, PEE and $\mathrm{PS}$ at $80^{\circ} \mathrm{C}, a=0.73$ for all three polymers. We have estimated that $K_{\mathrm{PE}}=1.7 K_{\mathrm{PEE}}=4.1 K_{\mathrm{PS}}$ based on published results obtained under theta and good solvent conditions, ${ }^{8}$ and calculated corrected molecular weights assuming $K_{\mathrm{PE}-\mathrm{PEE}}$ $=f_{\mathrm{PE}} K_{\mathrm{PE}}+f_{\mathrm{PEE}} K_{\mathrm{PEE}}$. The results are listed in Table I. Within experimental error and the uncertainties in these corrections, the modified GPCbased molecular weights are essentially equal, with an average value, $\left\langle M_{n}\right\rangle=83 \mathrm{~kg} / \mathrm{mol}$ that is almost identical to the one derived from the synthesis stoichiometry, $M_{n}=84 \mathrm{~kg} / \mathrm{mol}$ for all compounds.

PEE and PE are characterized by glass transition and melting temperatures at $-20^{\circ} \mathrm{C}$ and $108^{\circ} \mathrm{C}$, respectively, with a $\mathrm{PE}$ percent crystallinity of approximately $40 \%$ at room temperature. ${ }^{9}$

\section{Sample Preparation-Precipitation}

Samples were prepared using a precipitation or solvent casting technique. The precipitation technique is as follows: a heated $\left(>70^{\circ} \mathrm{C}\right)$ solution of PE-PEE in cyclohexane (pure diblocks) or toluene (blends) was poured into a beaker containing methanol (a nonsolvent for PE-PEE) at room temperature. This created a $2.5: 1$ solution of methanol to solvent. The high molecular weight diblocks immediately precipitated and the excess solvent was decanted. The polymer was collected on a filter and dried at elevated temperatures in a vacuum oven to remove the residual solvent. Uniform films of $1 \mathrm{~mm}$ thickness were made by pressing the dried polymer between two Teflon sheets (with $1 \mathrm{~mm}$ spacers) at $120^{\circ} \mathrm{C}$ in a vacuum oven.

\section{Sample Preparation-Solvent Casting}

Solvent cast samples were prepared by casting a $10 \% \mathrm{w} / \mathrm{v}$ solution of polymer in decahydronaphthalene on glass slides at $140^{\circ} \mathrm{C}$ (the glass slides were treated with dichlorodimethylsilane as a release agent). The sample was held at $140^{\circ} \mathrm{C}$ subject to a constant nitrogen purge for $4 \mathrm{~h}$, and then dried under vacuum overnight. Samples were recovered by quenching the polymer coated high temperature glass slide in liquid nitrogen (the polymer is easily removed at liquid nitrogen temperatures) and allowing the polymer film to warm to room temperature. Thin solvent cast films were folded multiple times and pressed at elevated temperatures in a vacuum oven to form 1-mmthick sheets.

\section{Shear Alignment}

Pressed films were gently sheared in an oscillating parallel plate geometry to enhance the degree of long-range order of the microstructure ${ }^{9}$ which facilitates morphological assignment based on TEM images. Samples were sheared at $150^{\circ} \mathrm{C}$ under a nitrogen blanket for about $10 \mathrm{~h}$ (one sample, precipitated PE-PEE-23, was sheared for $49 \mathrm{~h}$ ) with a strain rate $|\dot{\gamma}|=0.065 \mathrm{~s}^{-1}$ and $100 \%$ strain amplitude. The samples were cooled quickly to 
$50^{\circ} \mathrm{C}$ with liquid nitrogen before they were removed from the shearing device, then quenched in a dewar containing liquid nitrogen. This process fixes the morphology present at $150^{\circ} \mathrm{C}$ through rapid crystallization of the $\mathrm{PE}$ domains.

Sheared samples were annealed in argon flushed and vacuum sealed glass ampoules for 6 days at $150^{\circ} \mathrm{C}$ using a temperature-controlled oil bath. One exception was PE-PEE-20 which was annealed for 4 weeks. Annealed samples were quenched from $150^{\circ} \mathrm{C}$ in liquid nitrogen to fix the ordered phase. ${ }^{4,10}$ Annealed samples showed no signs of degradation, as confirmed with high-temperature GPC.

\section{Transmission Electron Microscopy}

Specimens for TEM were prepared by microtoming at $-100^{\circ} \mathrm{C}$ using a diamond knife to obtain thin sections of $\sim 90 \mathrm{~nm}$ thickness. The thin sections were stained in the vapors of a $0.5 \%$ aqueous $\mathrm{RuO}_{4}$ solution which preferentially stains the PEE phase, providing sufficient contrast for TEM imaging. ${ }^{10}$ Images were obtained with a JEOL 1210 microscope using an accelerating voltage of $120 \mathrm{kV}$.

\section{Small-Angle X-Ray Scattering}

SAXS experiments were conducted with a custom-built system at the University of Minnesota utilizing a rotating anode generator, Franks mirror focusing optics, a wavelength of $\lambda=1.54 \AA$, and an area detector. SAXS data were collected at $150^{\circ} \mathrm{C}$ on the shear aligned and annealed pure diblock samples. The first-order reflection could not be resolved from the parasitic scattering, therefore the principal spacing was calculated from higher-order reflections assuming a morphology identified with TEM. Lamellar samples (PE-PEE-20 and PE-PEE-22) contained a higher order reflection at $3 q^{*}$ and hexagonally packed cylinder samples (PE-PEE-21 and PE-PEE-23) contained a higher-order reflection at $\sqrt{ } 3 q^{*}$, where $q=(4 \pi / \lambda) \sin (\theta / 2)$ is the magnitude of the scattering wavevector. The principal spacing, $d^{*}$, is calculated from $d^{*}=2 \pi / q^{*}$.

\section{RESULTS AND ANALYSIS}

\section{Pure Diblock Copolymers}

The phase behavior of the four pure diblocks was characterized with transmission electron micros-

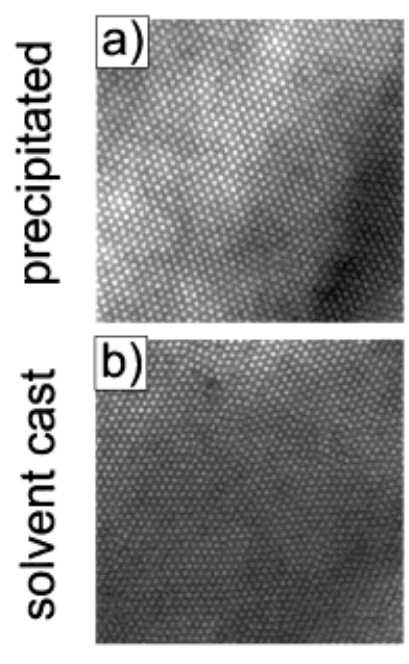

\subsection{5}
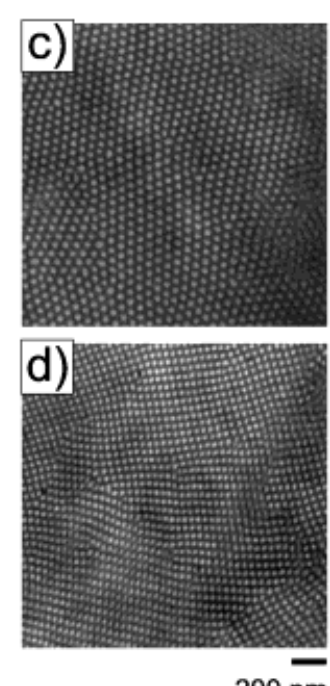

$200 \mathrm{~nm}$

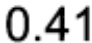

Figure 2. "Axis view" TEM images from PE-PEE-21 $\left(f_{\mathrm{PE}}=0.35\right)$ (a) precipitated, and (b) solvent cast, and from PE-PEE-23 ( $\left.f_{\mathrm{PE}}=0.41\right)$, (c) precipitated, and $(\mathrm{d})$ solvent cast. The PEE matrix is preferentially stained black by $\mathrm{RuO}_{4}$ and the hexagonally packed PE cylinders appear white.

copy (TEM) and small-angle X-ray scattering (SAXS). Samples prepared using both the precipitation and solvent casting techniques were imaged with TEM after shear alignment and annealing. Since the annealed samples were quenched from $150^{\circ} \mathrm{C}$ using liquid nitrogen, we assume the TEM images are representative of the phase behavior at $150^{\circ} \mathrm{C} .{ }^{4,10}$ The TEM images presented in Figures 2 and 3 are all "axis views" in the direction of the shear velocity as shown in Figure 1.

Given the volume fraction of the four pure diblocks and where they map onto the phase diagram in Figure 1, it is expected a priori that these diblocks will form either cylindrical, lamellar, or complex microstructures. Oscillatory shear will align cylinder samples with their axis parallel to the shear velocity. ${ }^{9}$ Thus, when they are imaged in the TEM along the shear direction, circular cross-sections of the cylinders are observed packed on a hexagonal lattice surrounded by the matrix material. Lamellae samples will orient in either a parallel or perpendicular orientation; for our particular shear conditions (well below $T_{\mathrm{ODT}}$ ), parallel lamellae are expected. ${ }^{11}$ A TEM image down the shear axis will view the layers edge on producing alternating dark and light stripes. Perforated lamellae will produce a similar image, but 
perforations should be evident in the minority component layers. If a gyroid morphology was to occur, it would produce a very distinctive TEM image with bicontinuous domains.

Axis view TEM images for PE-PEE-21 from precipitated (Fig. 2a) and solvent cast (Fig. 2b) samples both show white circles surrounded by a black matrix. Since the PEE is preferentially stained, it appears black while the PE appears white. Both the selective staining and the volume fraction of the sample, $f_{\mathrm{PE}}=0.35$, are consistent with a morphology of hexagonally packed PE cylinders in a PEE matrix. The cylinders are well aligned parallel to the shear direction. Grain boundaries and packing defects are readily identified with this axis view. Analogous results are obtained from the precipitated (Fig. 2c) and solvent cast (Fig. $2 \mathrm{~d}$ ) samples of $\mathrm{PE}-\mathrm{PEE}-23$ at $f_{\mathrm{PE}}$ $=0.41$.

The shear axis TEM images for PE-PEE-20 $\left(f_{\mathrm{PE}}=0.46\right)$ show different morphologies for the two preparation techniques. The precipitated sample (Fig. 3a) shows a striped image expected from a lamellar morphology, but close examination reveals light gray perforations in the white

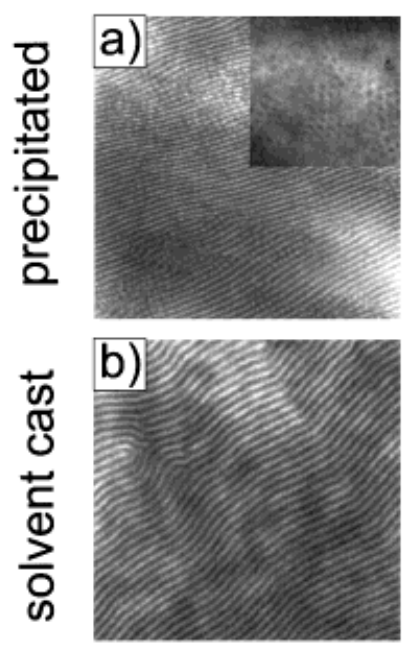

0.46
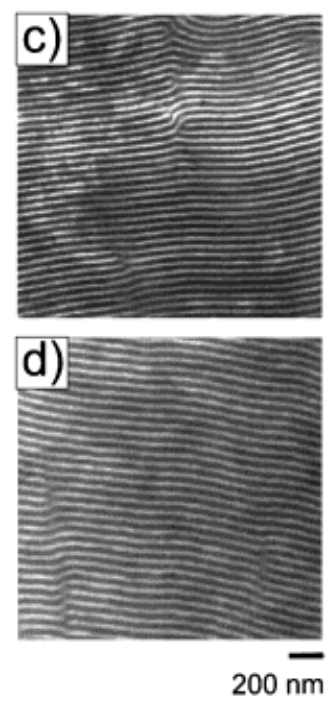

0.51
Figure 3. "Axis view" TEM images from PE-PEE-20 $\left(f_{\mathrm{PE}}=0.46\right)$ and PE-PEE-22 $\left(f_{\mathrm{PE}}=0.51\right)$. Perforations through the white $\mathrm{PE}$ layers are evident in some regions of the (a) precipitated PE-PEE-20 sample; the "axis view" normal to the layers (inset) shows that these perforations are hexagonally packed. The solvent cast sample (b) shows a classical lamellar structure. A well-aligned lamellar structure is obtained when PEPEE-22 is either (c) precipitated, or (d) solvent cast.

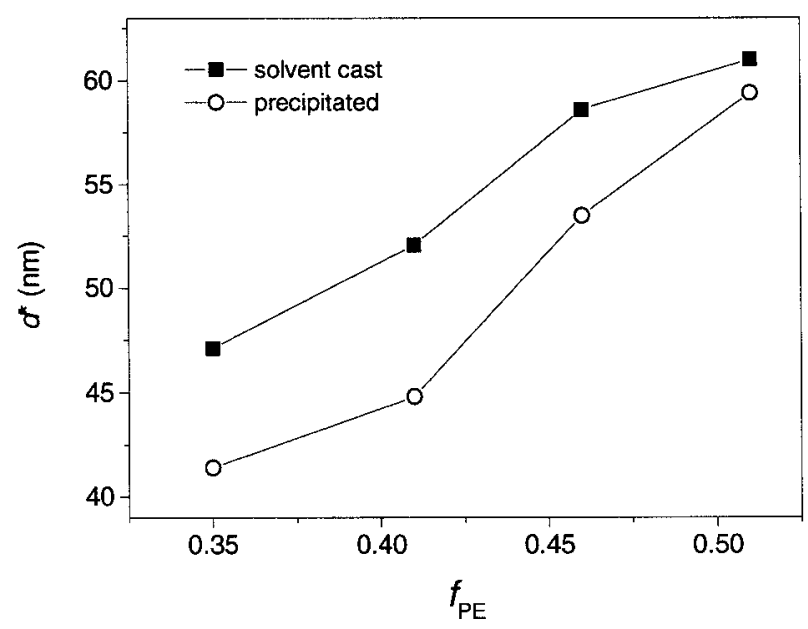

Figure 4. Principal spacings from the neat diblock melts measured for both preparation techniques using small-angle X-ray scattering.

PE layers. When viewed parallel to the layer normal (i.e., top view, Fig. 1), a uniform background with dark circles arranged hexagonally is observed (Fig. 3a, inset), which is consistent with PEE perforations through the PE layers. Figure $3 \mathrm{a}$ (axis view) also contains regions without any discernible perforations, and therefore the precipitated sample is identified as coexisting lamellae and perforated lamellae. On the other hand, the solvent cast sample (Fig. 3b) displays a wellaligned lamellar structure (grain sizes on the micron scale) void of any perforations.

Shear axis TEM images from PE-PEE-22 $\left(f_{\mathrm{PE}}\right.$ $=0.51$ ) prepared by precipitation (Fig. 3c) and solvent casting (Fig. 3d) both show well-aligned lamellae structures. Although shear alignment helps to organize the lamellae into large grains, structural defects are still evident. Notice the edge dislocations in Figure 3 where one layer terminates and the two neighboring layers merge.

Figure 4 shows the principal domain spacing for the four diblocks as measured by SAXS. A clear preparation dependence is evident; the spacings of the solvent cast samples are consistently larger than those of the precipitated samples. Differences range from $\sim 7.0 \mathrm{~nm}(\sim 17 \%)$ for $\mathrm{PE}-$ PEE-23 to $\sim 1.5 \mathrm{~nm}(\sim 3 \%)$ for PE-PEE-22. The large discrepancies $(>5 \%)$ are experimentally significant, and they persist even after substantial annealing times well above the glass-transition temperature.

\section{Binary Diblock Blends of PE-PEE-21 and PE-PEE-22}

Here we examine binary blends of PE-PEE-21 (cylinders, $f_{\mathrm{PE}}=0.35$ ) and PE-PEE-22 (lamellae, 


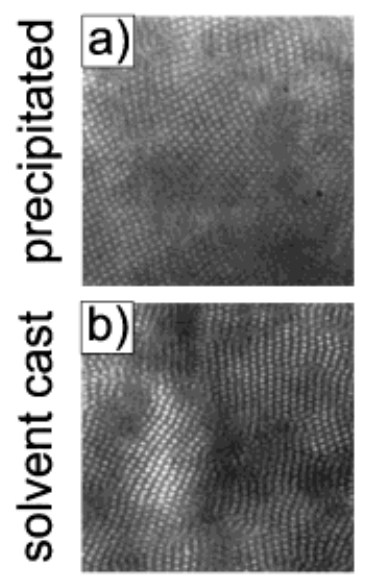

0.40
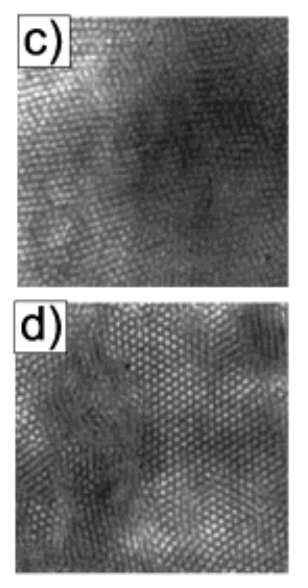

0.42
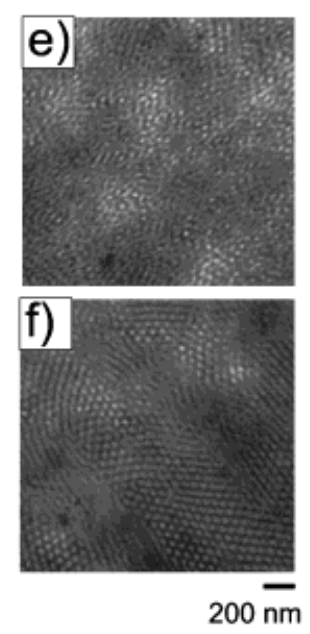

Figure 5. "Axis view" TEM images from binary blends of PE-PEE-21 and PEPEE-22 with $\left\langle f_{\mathrm{PE}}\right\rangle=0.40,0.42$, and 0.44 . Hexagonally packed PE cylinders in a PEE matrix are evident in each image, except (e) where a disordered bicontinuous structure occurs.

$\left.f_{\mathrm{PE}}=0.51\right)$ at $\mathrm{PE}$ volume fractions of $\left\langle f_{\mathrm{PE}}\right\rangle$ $=0.40,0.42,0.44,0.46,0.48$, and 0.50 . TEM images from the precipitated (Fig. 5a) and solvent cast (Fig. 5b) samples at $\left\langle f_{\mathrm{PE}}\right\rangle=0.40$, both show white circles on a black background consistent with PE cylinders in a PEE matrix. Hexagonal packing of the cylinders is evident in both images, but there are far more packing defects and diffuse grain boundaries than for the pure diblocks (Fig. 2 ). Analogous images are obtained for the precipitated (Fig. 5c) and solvent cast (Fig. 5d) blends at $\left\langle f_{\mathrm{PE}}\right\rangle=0.42$.

At $\left\langle f_{\mathrm{PE}}\right\rangle=0.44$, the TEM images display different morphologies depending on the sample preparation technique. The precipitated blend (Fig. 5e) has a microphase separated structure, but with disordered PE and PEE microdomains that both appear to be continuous; we refer to this structure as disordered bicontinuous. The solvent-casting technique (Fig. 5f) produces a cylinder structure, but with even more defects and grain boundaries than the $\left\langle f_{\mathrm{PE}}\right\rangle=0.40$ and 0.42 blends.

Preparation dependence is also evident in the $\left\langle f_{\mathrm{PE}}\right\rangle=0.46$ blend. Again the precipitated blend (Fig. 6a) displays a disordered bicontinuous structure. However, the solvent cast blend (Fig. 6b) produces a TEM image containing hexagonally packed circles, stripes, and numerous defects, consistent with a morphology of coexisting cylinders and lamellae.
The preparation dependence still persists at $\left\langle f_{\mathrm{PE}}\right\rangle=0.48$. The precipitated sample (Fig. 6c) again exhibits a disordered bicontinuous morphology, but the solvent cast blend (Fig. 6d) produces a lamellar morphology. Very few defects exist in the lamellar structure [i.e., the orientational order and the grain sizes are long-range (>1 micron)].

$\mathrm{By}\left\langle f_{\mathrm{PE}}\right\rangle=0.50$, the preparation dependence of the morphology is no longer evident, excluding possible subtle differences in lamellar spacing. Both the precipitated (Fig. 6e) and solvent cast (Fig. 6f) samples produce well-ordered lamellar structures.

The experimental phase behavior of the binary blends is summarized in Figure 7a for the solvent cast samples and Figure $7 \mathrm{~b}$ for the precipitated samples. (Recall that all our samples where annealed at $150^{\circ} \mathrm{C}$, which corresponds to $\chi N=45$ ). We compare these results to meanfield predictions ${ }^{5}$ based on the standard, incompressible, Gaussian-chain model $^{2}$ with a conformational asymmetry of $a_{\mathrm{PE}} / a_{\mathrm{PEP}}=1.5{ }^{12}$ (Note a common segment volume is used when calculating the statistical lengths, $a_{\mathrm{PE}}$ and $a_{\mathrm{PEP}}$.) Figure $7 \mathrm{c}$ shows the theoretical phase diagram obtained for a binary blend ( $f_{\mathrm{PE}}=0.35+f_{\mathrm{PE}}=0.51$ ) when only the cylinder and lamellae structures are considered. It corresponds well with the behavior of the solvent cast samples (Fig. 7a), but it is not the equilibrium phase diagram. The equilibrium dia- 


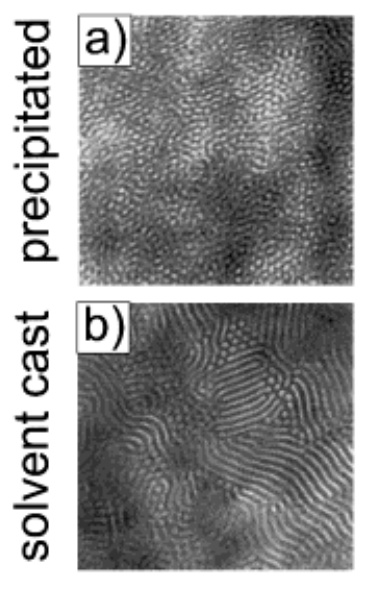

0.46
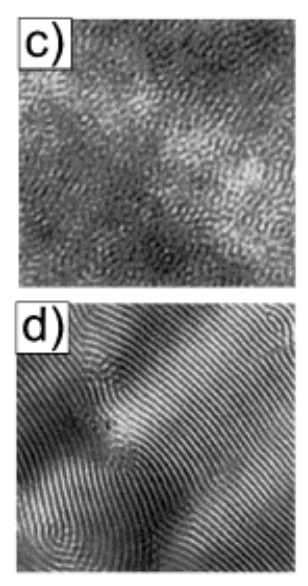

0.48
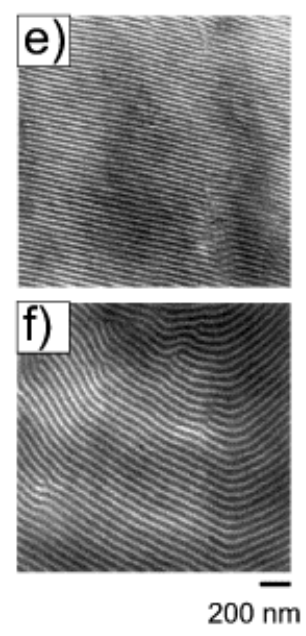

0.50

Figure 6. "Axis view" TEM images from binary blends of PE-PEE-21 and PEPEE-22 with $\left\langle f_{\mathrm{PE}}\right\rangle=0.46,0.48$, and 0.50 . The $\left\langle f_{\mathrm{PE}}\right\rangle=0.46$ and 0.48 samples form disordered bicontinuous structures, (a) and (c), when precipitated, but either coexisting cylinders and lamellae (b) or lamellae (d) when solvent cast. The $\left\langle f_{\mathrm{PE}}\right\rangle=0.50$ blend forms a lamellar morphology when precipitated (e) or solvent cast (f).

gram, obtained by mapping the lowest free energy states, includes a stability region for the gyroid phase as shown in Figure 7d. This diagram agrees well with the precipitated samples (Fig. 7b) provided we associate the disordered bicontinuous state with the gyroid phase. The fact the theoretical phase boundaries in Figure 7 are shifted to smaller $\left\langle f_{\mathrm{PE}}\right\rangle$ relative to the experimental ones indicates that the conformational asymmetry may be somewhat larger than $a_{\mathrm{PE}} / a_{\mathrm{PEP}}=1.5$.

Due to numerical limitations, ${ }^{1}$ the gyroid phase could only be examined accurately up to segregations of $\chi N \approx 35$. The dashed phase boundaries at the higher segregations in Figure $7 \mathrm{~d}$ are extrapolated from the $\chi N<35$ results. Although there will be some inaccuracy in our extrapolations, we believe that the gyroid phase remains stable well beyond $\chi N=45$.

\section{DISCUSSION}

The phase identification from TEM images for the four pure diblocks and the six binary blends of PE-PEE-21 and PE-PEE-22 are summarized in Table I and Figure 7, respectively. The pure diblock PE-PEE-20 (Fig. 3a and b) exhibited different morphologies for two preparation techniques even after 4 weeks of annealing. Similarly, the blends at $\left\langle f_{\mathrm{PE}}\right\rangle=0.44,0.46$, and 0.48 (Figs. 5e and $f$ and $6 a-d)$ each exhibited different morphologies after 6 days of annealing. This preparation dependence, despite prolonged annealing, demonstrates that these high molecular weight ( $\left.M_{n} \cong 84 \mathrm{~kg} / \mathrm{mole}\right)$, intermediately segregated ( $\chi N \cong 45$ ) diblock copolymers have great difficulty reaching an equilibrium state. If equilibrium had been achieved, the morphology would depend only on the final state (i.e., temperature, pressure, and composition) and not at all on the sample preparation. The same applies to the domain spacing, and thus the preparation dependence evident in Figure 4 is another example of nonequilibrium behavior. Furthermore, the precipitated PE-PEE-20 sample showed evidence of phase coexistence (lamellae with perforated lamellae), which is strictly prohibited for a monodisperse pure melt in equilibrium, except at an order-order transition temperature.

The two preparation techniques produce very different starting conditions. In both cases, the sample is first dissolved in a dilute high temperature solution where the unfavorable interactions between the PE and PEE are screened by the solvent, resulting in a homogeneous or disordered solution. However, the solution is removed in two very different ways. A precipitated sample is rapidly recovered by pouring the high temperature solution into a room-temperature nonsolvent. Since the material precipitates immediately and 


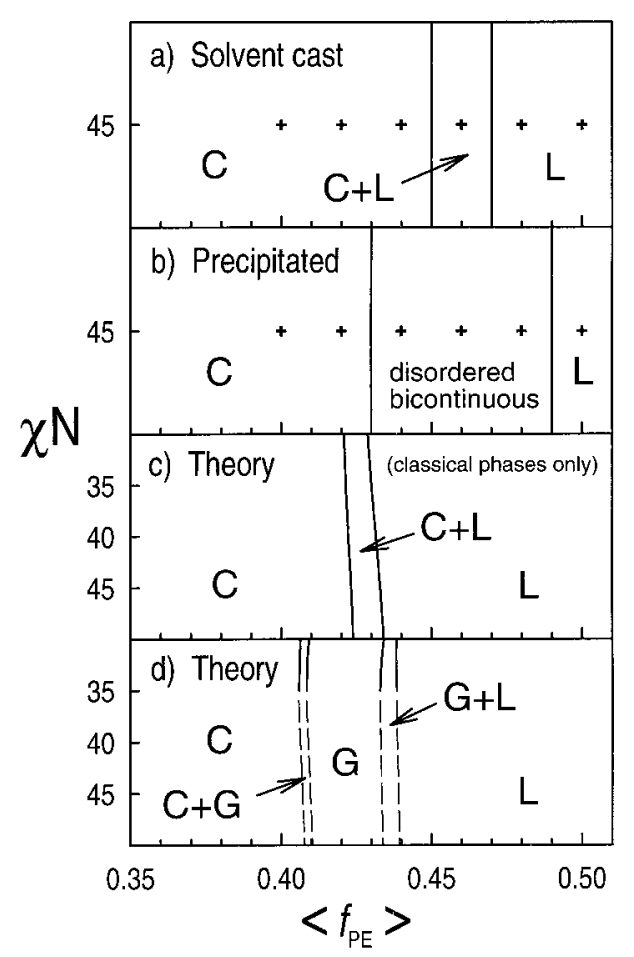

Figure 7. Comparison of the experimentally determined blend phase behavior with mean-field theory predictions. Between the cylinder (C) and lamellae (L) regions, the solvent cast blends (a) exhibit a $\mathrm{C}+\mathrm{L}$ coexistence window, whereas the precipitated blends (b) exhibit a disordered bicontinuous state. (The $+\mathrm{s}$ denote the blends examined in this study.) When the complex phases are ignored, the SCFT theory predicts a phase diagram (c) like that of the solvent cast blends. However, when the complex phases are included, a gyroid (G) phase is predicted between $\mathrm{C}$ and $\mathrm{L}(\mathrm{d})$. This indicates that the precipitated samples are closer to equilibrium provided we consider the disordered state as a gyroid phase that has had insufficient time to develop long-range order. (The dashed phase boundaries are extrapolations from $\chi N<35$ )

the PE crystallizes, the system has insufficient time to form an ordered structure and instead it assumes a microphase separated disordered state much like that in Figures 5e and 6a and c. This was confirmed by examining unsheared samples by TEM. In contrast, solvent cast samples are recovered slowly. Since the casting occurs above the melt temperature, there is no concern of $\mathrm{PE}$ crystallization locking in the structure. Instead, as the solvent evaporates slowly, the solution becomes more concentrated and the interactions between PE and PEE are gradually increased allowing time for some degree of order to set in. Again this was supported by TEM images from unsheared samples.
The final stage of both preparation techniques involved a gentle oscillatory shear applied in order to break the orientational degeneracy of the sample. By doing so, grains of the ordered phase are expected to grow with similar orientations allowing the sample to develop long-range order more easily. It may be that the shear provides a preference to a metastable morphology (i.e., perforated lamellae over gyroid), which may actually increase the annealing time required to reach equilibrium. Nevertheless, our concern is how long do samples have to be annealed before equilibrium is achieved, and to assess this we just need to compare samples with different initial conditions. Certainly, our two preparation methods have accomplished this.

One factor contributing to the nonequilibrium behavior observed in our study is the low chain mobility resulting from the high molecular weight and the large degree of segregation. There are two modes of chain diffusion, which have been studied by Lodge and co-workers ${ }^{13,14}$ using PEP-PEE diblock copolymers. Parallel diffusion occurs when block copolymers move with their junctions constrained to the interface. When $\chi N>20$, this mode is controlled by the number of chain entanglements $\left(N / N_{e}\right.$, where $N_{e}$ is the entanglement chain length). Since our PE-PEE diblocks have a similar number of entanglements $\left(N / N_{e} \sim 40\right)$ as the PEP-PEE diblocks in Ref. 13, we can expect a similar parallel diffusion constant. The other mode, perpendicular diffusion, is a function of $\chi N$ because it involves one block diffusing through the domain of the other block. The results of Lodge and co-workers ${ }^{13}$ for PEP-PEE at $\chi N=45$ suggest that this mode is particularly slow, in fact five orders of magnitude slower than diffusion in an unstructured matrix. Thus, we can attribute part of the metastability experienced in our study to slow perpendicular diffusion.

A second factor contributing to the metastability of our PE-PEE samples is their proximity to a phase boundary. ${ }^{15}$ Deep in a one-phase region, there is a strong tendency to form the equilibrium morphology because its energy should be much lower than that of other morphologies. This is no longer true near a phase boundary, particularly the cylinder/lamellae boundary where complex phases also compete for stability. Consistent with this, the samples where the morphology was preparation dependent were those close to the phase boundary. Furthermore, the long-range order diminished as the boundary was approached. 
Other recent studies have identified persistent metastability near the cylinder/lamellae phase boundary in pure diblock copolymer melts. ${ }^{16}$ There the perforated layer phase was shown to be a long-lived nonequilibrium morphology that eventually transforms to the gyroid morphology. This was the case for low molecular weight PEPEEs near the ODT ( $\left.M_{n} \cong 40 \mathrm{~kg} / \mathrm{mole}\right)$, and may also be true for PE-PEE-20, although this has not been confirmed. The metastability of perforated lamellae occurs presumably because its energy is so close to that of gyroid, ${ }^{17}$ which in turn is because the minority domains of both structures are constructed from similar threefold coordinated elements.

It is possible that one of our preparation techniques produced equilibrium behavior while the other produced long-lived nonequilibrium states, but we suspect that both resulted in nonequilibrium behavior. It is very unlikely that the disordered bicontinuous state observed in the precipitated blends could be equilibrium at high degrees of segregation where fluctuation effects are presumably negligible. The theory suggests that the true equilibrium phase is gyroid. Because the disordered bicontinuous state is structurally similar to gyroid, we suggest the energy difference was simply insufficient to develop long-range order in the time scale of the experiment. Based on the theoretical predictions, the solvent cast samples were furthest from equilibrium even though they displayed a higher degree of order. We suggest that their morphology reflects an equilibrium state during the early stages of solvent casting, where cylinder + lamellae coexistence is favored over gyroid. Presumably, gyroid becomes stable as the solvent evaporates, but by then the reduced chain mobility locks in the cylinder + lamellae coexistence.

Also interesting is the fact that the disordered bicontinuous state found in the binary mixtures at $0.43<\left\langle f_{\mathrm{PE}}\right\rangle<0.48$ is not seen in the $f_{\mathrm{PE}}$ $=0.46$ single component specimen. Clearly, mixing two diblocks, with two different compositions, does not duplicate the molecular packing conditions that characterize a single component diblock melt. Apparently, nonequilibrium processing histories can exacerbate the differences. One possibility is that local (i.e., domain scale) segregation of different block copolymers could stabilize the interfacial curvature that dominates the disordered bicontinuous state. Whether this is actually an equilibrium morphology, or simply a local minimum in the global free energy cannot be established at this time. We suspect that this structure is close in free energy to the gyroid phase, which the SCFT theory anticipates (Fig. 7 ), which could make nucleation and growth of order prohibitively slow. Regardless of the true thermodynamic state of these specimens, the blending protocal appears to be a reliable strategy for producing a random bicontinuous morphology which could have useful applications.

Independent of any interpretation, this work has clearly demonstrated pronounced metastability and nonequilibrium phase behavior in intermediately segregated block copolymers. These results highlight the care that must be used when reporting the phase behavior away from the ODT, ${ }^{18}$ particularly near order-order phase boundaries, and when dealing with blended specimens. Monomodal polydispersity may also play a contributing role although we have not examined that situation. These difficulties in attaining equilibrium will likely be exacerbated in the more complicated multiblock copolymers (ABA, ABAB, $\mathrm{ABC}$, etc.), that are frequently studied at high degrees of segregation. ${ }^{19}$

\section{SUMMARY}

The phase behavior of four pure PE-PEE diblock copolymers and six binary diblock blends in the intermediate segregation regime $(\chi N=45)$ was characterized using transmission electron microscopy and small-angle X-ray scattering. Samples were prepared by either solvent casting or precipitation followed by gentle shearing to break the orientational symmetry and then lengthy annealing high above the glass transition. Despite the extensive annealing, solvent cast and precipitated samples did not attain a common equilibrium state. One of the pure melts and three of the blends displayed different morphologies dependent on the initial preparation technique. Precipitation of a binary mixture of symmetric and asymmetric diblock copolymers led to the formation of a uniform disordered bicontinuous morphology, while solvent casting the same blends induced phase separation. Furthermore, the domain spacing measured for the pure melts from solvent cast and precipitated samples differed by up to $17 \%$. This nonequilibrium behavior is attributed to the high degree of segregation and the close proximity to the cylinder/lamellae phase boundary. 
FSB received support for this work from the Air Force Office of Scientific Research under contract AF/F4962096-1-0088, and MWM was supported by a grant from the Nuffield Foundation.

\section{REFERENCES AND NOTES}

1. Matsen, M. W.; Bates, F. S. Macromolecules 1996, 29, 1091.

2. Rosedale, J. H.; Bates, F. S.; Almdal, K.; Mortensen, K.; Wignall, G. Macromolecules 1995, 28, 1429 .

3. Bates, F. S.; Schulz, M. F.; Khandpur, A. K.; Forster, S.; Rosedale, J. H.; Almdal, K.; Mortensen, K. Royal Chem Soc, Faraday Trans 1994, 98, 1.

4. Zhao, J.; Majumdar, B.; Schulz, M. F.; Bates, F. S.; Almdal, K.; Mortensen, K.; Hajduk, D. A.; Gruner, S. M. Macromolecules 1995, 29, 1204.

5. Matsen, M. W.; Bates, F. S. Macromolecules 1995, 28, 7298.

6. Khandpur, A. K.; Förster, S.; Bates, F. S.; Hamley, I. W.; Ryan, A. J.; Bras, W.; Almdal, K.; Mortensen, K. Macromolecules 1995, 28, 8796; Schulz, M. F.; Khandpur, A. K.; Bates, F. S.; Almdal, K.; Mortensen, K.; Hajduk, D. A.; Gruner, S. M. Macromolecules 1996, 29, 2857; Zhao, J.; Majumdar, B.; Schulz, M. F.; Bates, F. S.; Almdal, K.; Mortensen, K.; Hajduk, D. A.; Gruner, S. M. Macromolecules 1995, 29, 1204; Almdal, K.; Rosedale, J. H.; Bates, F. S. Macromolecules 1990, 23, 4336; Sakurai, S.; Irie, H.; Umeda, H.; Nomur, S.; Lee, H. H.; Kim, J. K. Macromolecules 1998, 31, 336; Almdal, K.; Mortensen, K.; Ryan, A. J.; Bates, F. S. Macromolecules 1996, 29, 5940.

7. Fetters, L. J.; Lohse, D. J.; Richter, D.; Witten, T. A.; Zirkel, A. Macromolecules 1994, 27, 4639.
8. Polymer Handbook; John Wiley \& Sons: New York, 1989; 3rd ed.

9. Khandpur, A. K.; Macosko, C. W.; Bates, F. S. J Polym Sci Part B: Polym Phys 1995, 33, 247.

10. Koppi, K. A.; Tirrell, M.; Bates, F. S.; Almdal, K.; Mortensen, K. J Rheol 1994, 38, 999.

11. Koppi, K. A.; Tirrell, M.; Bates, F. S.; Almdal, K.; Colby, R. H. J Phys II France 1992, 2, 1941.

12. Matsen, M. W.; Bates, F. S. J Polym Sci Part B: Polym Phys 1997, 35, 945.

13. Lodge, T. P.; Dalvi, M. C. Phys Rev Lett 1995, 75, 657.

14. Lodge, T. P.; Hamersky, M. W.; Milhaupt, J. M. Macromol Symp 1997, 121, 219.

15. Gido, S. P.; Wang, Z.-G. Macromolecules 1997, 30, 6771.

16. Hajduk, D. A.; Takenouchi, H.; Hillmyer, M. A.; Bates, F. S.; Vigild, M. E.; Almdal, K. Macromolecules 1997, 30, 3788.

17. Matsen, M. W.; Bates, F. S. J Chem Phys 1997, 106, 2436.

18. Winey, K. I.; Thomas, E. L.; Fetters, L. J. Macromolecules 1991, 24, 6182; Disko, M. M.; Liang, K. S.; Behal, S. K.; Roe, R. J.; Jeon, K. J. Macromolecules 1993, 26, 2983; Sakurai, S.; Hashimoto, T.; Fetters, L. J. Macromolecules 1995, 28, 7947; Koizumi, S.; Hasegawa, H.; Hashimoto, T. Macromolecules 1994, 27, 7893; Torikai, N.; Takabayashi, N.; Noda, I.; Koizumi, S.; Morii, Y.; Matsushita, Y. Macromolecules 1997, 30, 5698; Shibayama, M.; Hashimoto, T.; Hasegawa, H.; Kawai, H. Macromolecules 1983, 16, 1427.

19. Isono, Y.; Tanisugi, H.; Endo, K.; Fujimoto, T.; Hasegawa, H.; Hashimoto, T.; Kawai, H. Macromolecules 1983, 16, 5; Stadler, R.; Auschra, C.; Beckmann, J.; Krappe, U.; Voight-Martin, I.; Leibler, L. Macromolecules 1995, 28, 3080; Breiner, U.; Krappe, U.; Thomas, E. L.; Stadler, R. Macromolecules 1998, 31, 135. 